\title{
IL CIBO FRA NATURA E CULTURA: ALCUNI ASSAGGI DI POESIA GASTRONOMICA GRECA
}

\author{
ORNELLA MONTANARI (*) \\ Gli animali si sfamano, l'uomo mangia, solo l'uomo d'ingegno sa mangiare. \\ (A. Brillat-Savarin, II Aforisma)
}

SunTO. - Un oscuro autore greco, in genere ignorato dalle Storie della letteratura, Archestrato di Gela, è ritenuto dai critici più qualificati il "Father of Western Gastronomy". Egli operò nella seconda metà del IV sec. a.C.: il suo poema, Hedypatheia (Vita di delizie), è un singolare Lehrgedicht, frutto di un'arte raffinata, sorvegliatissima ma mai stucchevole, piena di bumour, che scaturisce dall' inatteso contrasto tra l'austera forma esametrica e il singolare contenuto gastronomico. Un prodotto serio-comico di sicuro impatto allusivo. Ne conosciamo in tutto 62 frammenti, per un totale di circa 330 versi. L' antico gastronomo ha girato il mondo per philedonia e si è divertito a trascrivere le sue gustose esperienze, nella ferma convinzione che solo la diretta autopsia possa essere garanzia di successo in cucina. Una sorta di "breviario del buongustaio" (Lesky) che dà luogo alla legge del DOVE/COME/QUANDO, sicuro viatico, anche ai giorni nostri.

$$
* * *
$$

ABSTRACT. - A little known Greek author, generally ignored by most handbooks of literary history, Archestratos of Gela, is regarded by the most qualified scholars as the "Father of Western Gastronomy". He worked during the second half of the $4^{\text {th }}$ century BC; his poem, Hedypatheia (Life of pleasure), is a peculiar Lebrgedicht, result of a refined art, very well controlled and never excessive, humourous, resulting from an unexpected contrast between the hexametric form and the original grastronomic topic. From this serio-comic and allusive work only 62 fragments survive, amounting around 330 lines. This ancient gastronome travelled all around the world for the sake of philedonia and had much fun translating his taste experiences into verse. He thought that only direct autopsy is a guarantee of success in cooking. This is a sort of "vademecum of the gourmet" (Lesky), that generates the rule of WHERE/HOW/WHEN, which is still an excellent guide.

(*) Università degli Studi di Bologna, Italia. E-mail: ornella.montanari@unibo.it 
La vulgata telematica, che vede in Wikipedia lo strumento di immediata alfabetizzazione, individua, pur con qualche esitazione, nella Fisiologia del gusto di Jean Anthelme Brillat-Savarin «il primo trattato di gastronomia».

In realtà l'erudito magistrato, consigliere di Cassazione nonché autore di numerosi saggi di carattere giuridico-economico, mai avrebbe immaginato che la sua fama restasse ancorata a quell'oscuro libretto, pubblicato anonimo nel 1825, un anno prima della sua scomparsa. Non si trattava - per ammissione del compilatore stesso - di «un semplice libro di cucina» bensì di una riflessione globale sui piaceri della tavola e sui benefici influssi che il cibo esercita sulla vita e la felicità degli individui, sul successo o meno delle loro intraprese e dei loro affari.

Curiosamente, uguali intenti si era prefisso un autore greco di età ellenistica (IV/III sec. a.C.) ignoto ai più, Archestrato di Gela, la cui opera fu denominata secondo alcuni testimoni Gastronomia o Gastrologia, secondo altri Hedypatheia, vale a dire Vita di delizie. Lo stesso Brillat-Savarin non ignora il dimenticato progenitore, ma si appella all'autorità di un non meglio noto Teotimo per tracciarne un sommario profilo, in assenza - egli dice - di testimonianze dirette, disgraziatamente perdute. Le cose non stanno proprio così e cercheremo di dimostrarlo nei fatti. Avremo modo di leggere alcuni dei 62 frammenti di Archestrato giunti fino a noi, grazie alle cure di Ateneo di Naucrati (II sec. d.C.), che li cita con grande rispetto nel corso del VII libro dei Sofisti a banchetto, dedicato per intero ai pesci.

Il nostro autore si fa, a più riprese, cantore del lusso e della liberalità in cucina, raccomanda spirito d'iniziativa nella ricerca delle prelibatezze, indagine sul campo (autopsia), e soprattutto dovizia di mezzi. Non è il cantore delle mense plebee, ma un raffinato gourmet (opsologos), quanto mai preoccupato del decoro e della forma, attento interprete di un misurato stile di vita.

Se è vero che gli ultimi 30 anni del secolo scorso hanno visto l'accentuarsi dell'interesse per generi cosiddetti minori della letteratura greca, è ancora più vero il polarizzarsi dell'attenzione sulla poesia parodico-gastronomica. Nel precedente disinteresse aveva pesato moltissimo il pre-giudizio, nei confronti di una produzione ritenuta bumilis e di limitato pregio poetico.

A ragione Enzo Degani affermava: «Il comico è sempre stato tenuto in subordine in quanto 'futile' rispetto al 'serio' e la parodia, in quanto genere parassitario o che vive sulle spalle di un altro genere più 
nobile - la poesia epica - ha avuto di solito una vita grama, non solo in Grecia»1.

Quasi tutti i manuali di Letteratura Greca ignorano il tema o ad esso riservano qualche riga fuggevole, per lo più inficiata di grossolanità ed inesattezze di ogni genere. Tale presa di posizione non è che il riflesso di una latitanza generalizzata della critica sull'argomento e non c'è da meravigliarsi se si scopre che la voce Parodia manca nella monumentale e canonica «Enciclopedia Italiana» curata da Ernesto Treccani, e non solo nel volume di riferimento - come suole dirsi sub voce - che è del 1935, periodo di crocianesimo imperante, ma anche nei vari «Supplementi» successivi. Chi cerca la voce Parodia nell'Indice viene rinviato a Caricatura (redatta, peraltro, dall'archeologo Giovanni Patroni in collaborazione con Odoardo Hillyer Giglioli) o a Ironia, mirabilmente uscita dalla penna di Manara Valgimigli, che reca solo fuggevoli cenni al complesso meccanismo parodico.

Enzo Degani, dunque, è stato il primo, già agli albori degli anni '70, ad affrontare seriamente e sistematicamente il tema, concentrandosi sull'accezione più tecnica e specifica di tale prodotto poetico, vale a dire la detorsio Homeri ${ }^{2}$. Egli ebbe il merito di riportare alla luce e consegnare a nuova vita autori dimenticati, quali Archestrato di Gela e Matrone di Pitane, che divennero argomento di vari corsi monografici dell'Ateneo bolognese e di numerosissime tesi di laurea: si era aperto inopinatamente un nuovo fronte di ricerca ${ }^{3}$.

Con gli anni '90 del XX secolo tutto cambia radicalmente e vengono meno le preclusioni manifestate in passato verso questo tipo di produzione poetica.

Non solo l'universo legato al cibo diviene sempre più oggetto di attenzione e di ricerca, ma si apre il proficuo filone degli studi di antropologia storica sull'argomento: da Exeter a New York prendono piede ricerche pertinenti il tema 'gastronomico' e la bibliografia specifica diventa ben presto bibliografia di 'genere'. Anche come fatto letterario si dischiudono prospettive nuove, legate essenzialmente a due fattori,

1 Degani 1991, 155.

2 Degani 1973/1974, 141-167; Id. 1975, 157-174; Id. 1982, 29-54; Id. 1982/1983, 93-112.

3 Quasi inesistente la bibliografia su Archestrato, ad eccezione naturalmente dell'edizione critica dei frammenti a cura di Ribbeck 1877 e successivamente di Brandt 1888, 114-193. Di scarsa rilevanza il contributo di Alessandro Olivieri 1939, 110-117, 
che hanno dilatato le strettoie dell'analisi meramente erudita (che in passato aveva dominato il settore, confinandolo in una condizione di semi-oblio):

- la rivalutazione del comico, con la riscoperta bachtiniana del ruolo capitale che la parodia esercita nello sviluppo della storia d'Europa; - l'esplosione delle ricerche sull'intertestualità, con il conseguente approfondimento del concetto di 'memoria poetica', di 'intenzionalità' dell'autore.

Gli studi di Degani maturano vieppiù in questa bumus di rinnovamento, dopo una lunghissima incubazione che affonda le radici nell'Ipponatte parodico (1973/1974) e nelle Note ai parodi greci (1975). Il punto dottrinario non è mai fine a sé stesso, ma si fa strumento ermeneutico naturaliter. Quanto egli aveva scritto sulla «marina Cariddi» dell'Eurimedontiade e sulla panciaspada, che tutto tritura (Hippon. fr. 126) aveva aperto la strada ad una metodologia d'indagine che è, al tempo stesso, linguistica e filologica senza trascurare il vento nuovo, che - è bene ribadire - vede nell'italica terra il protos euretes.

Spiace che gli ultimi editori oxoniensi del poemetto archestrateo citino, nella essenziale bibliografia (p. XV), solo i primissimi lavori del compianto Maestro (le succitate Note ai parodi greci del 1975 e La poesia parodica greca del 1983), passando sotto silenzio i contributi fondamentali, che hanno avuto il merito di imporre all'attenzione degli studiosi questo particolarissimo genere ${ }^{4}$.

$\mathrm{Ma}$ è tempo di ritornare alla poesia gastronomica e al nostro autore, per degnamente celebrare «il padre della gastronomia occi-

mentre data al 1823 la prima traduzione in lingua italiana de I frammenti della Gastronomia, corredata da un breve commento. Ne è autore Domenico Scinà (1765 1837), illustre conterraneo siculo e fisico di vaglia, che aveva studiato dapprima «grammatica e umane lettere» nelle Scuole Pie, indi «filosofia, geometria e fisica» nella Reale Accademia degli Studi panormitana, infine «teologia», mirabile percorso degli eruditi settecenteschi! Tale pregevole lavoro sarà riproposto anastaticamente nel 1993 per celebrare i 40 anni dell'Accademia Italiana della cucina (fondata da Orio Vergani il 29 luglio 1953), in una serie limitata di soli 500 esemplari. Ancora una volta l'iniziativa nasceva con la benedizione di Enzo Degani, a cui si deve un'ampia nota introduttiva (pp. 9-51) e per iniziativa del barone Giuseppe Starrabba di Ralbiato, che aveva avuto modo di 'ripescare' fra i libri della propria biblioteca il prezioso volumetto, che l'aveva colpito per «l'attualità delle cose che l'autore diceva e il modo in cui le diceva» (Prefazione, p. 7).

4 Olson-Sens 2000. 
dentale», secondo la felice formula di Albert Rapp 5 . Prima il genere, dunque.

Si tratta di un tipico prodotto di 'intrattenimento', gradevole quanto sorridente, un lusus, certamente meno ingenuo di quanto si potrebbe a prima vista ipotizzare e, non di rado, frutto di un impegno artistico considerevole. Una poesia faceta e sorridente (ludibunda), che rientra nel genere ampio dello spoudogeloion, rivestendo di aulici panni un argomento umile, a volte triviale, con immediata e programmatica rottura del codice espressivo 'alto'.

Discorso a parte merita la giambografia. Ipponatte ama parlare di raffinate vivande, che mettono in campo elaborate salse di tonnina, formaggio, aglio ed aceto $(=\text { myssoton })^{7}$ oppure di francolini di monte, lepri e maritozzi al formaggio ${ }^{8}$, mentre Ananio, poeta giambico del VI a.C., sciorina un vero e proprio 'calendario gastronomico', che combina luogo di elezione del cibo e stagione propizia per fruirne, primo esempio di programmatica poesia edifagetica?.

Tale attenzione dilagherà nella commedia, soprattutto nell'Archaia, aprendo le porte alla «versione plebea dell'aristocratica

5 Rapp 1955, 43-48.

6 Non va dimenticato che il riferimento al cibo e alle funzioni corporali, agli organi sessuali e all'esternazione degli affetti risulta volutamente bandito dall'epica omerica, con una sorta di pruderie, che non deve ritenersi casuale. Gli eroi stessi o le donne preparano sommariamente il pasto, senza alcuna concessione ai dettagli, tutt'al più con qualche compiacenza 'rituale'. Illuminante, al proposito, Kerényi 1979. Manca la figura del cuoco di professione, come quella del parassita, che tanta parte avrà nel filone comico.

7 Hippon. fr. $26 \mathrm{Dg}$. «uno di loro placidamente e in abbondanza sbafandosi per tutto il giorno tonnina / e salsa piccante di formaggio, aglio e aceto, / come un eunuco di Lampsaco finì per divorarsi tutti gli averi, / e così deve zappare pietre montane, / cibandosi di fichi di modesta pezzatura e di pane d'orzo, cibo da schiavi».

8 Hippon. fr. 26a Dg. «divorando non francolini di monte né lepri, / temprando nella salsa di sesamo non maritozzi al formaggio, / né intingendo nel miele esotiche frittelline».

9 Anan. fr. 5 W. ${ }^{2}$ «In primavera ottimo è il cromio, d'inverno il serrano, / ma fra le buone pietanze la migliore è la caride, presa dalla sua foglia di fico. / Piacevole poi d'autunno gustare carne di capretto. / Ma quando si vendemmia e si pigia l'uva, questo è il momento di mangiar carne di porco,/ nonché di cane, di lepre e di volpe. / La pecora, in piena estate, quando friniscono le cicale. / Il tonno di mare non è cibo malvagio,/ ma eccelle su tutti gli altri pesci in salsa piccante. / Il bue ingrassato non ha stagioni, a mio parere, di giorno / come nel bel mezzo della notte». Cf. Ath. VII 282ab: «Ho citato parecchi versi di Ananio nel pieno convincimento che sia stata sua ferma intenzione disporre tali ammonimenti ad uso e consumo dei crapuloni». 
età dell'oro», che è il mito popolare del paese di cuccagna, vero leitmotif di tutta la Commedia di mezzo ${ }^{10}$.

$\mathrm{Si}$ incontrano di continuo pantagrueliche descrizioni di interminabili banchetti (Anaxandr. fr. 41 K.-A.) oppure sussiegose 'tirate' di cuochi, che esaltano le loro mirabolanti virtù davanti ai fornelli (Ephipp. fr. 8 K.-A.). È una documentazione imponente, che ci porterebbe troppo lontano. Ciò che preme sottolineare è che nella commedia il richiamo al ventre come quello al sesso - altra incoercibile manifestazione di forza vitale - deve dirsi di primaria rilevanza, ma si tratta, pur sempre, di tirate gastronomiche, inserite in opere di statuto epistemologico del tutto differente.

La letteratura specifica e di contenuto tecnico, sviluppatasi già a partire dalla seconda metà del V secolo, restò ostinatamente confinata in una produzione in prosa, di scarso valore artistico, oggi completamente perduta. Labili tracce di essa permangono nella farraginosa silloge di Ateneo di Naucrati, i cui Sofisti a banchetto debbono dirsi l'estrema propaggine antiquaria di una galassia di notevolissime dimensioni e di robusta e consolidata tradizione.

Niente o poco a che vedere con i veri maîtres à penser, di cui noi oggi celebriamo i fasti. Ed Archestrato di Gela (vissuto a cavallo fra IV e III secolo a.C.) fu uno di questi.

Girò il mondo per documentarsi su «Qual è e di quale specie, il pesce più gradevole / o qual è nella migliore stagione e /ancora qual è commestibile dopo Arturo e le Pleiadi o l'astro del Cane» (fr. 3 OlsonSens). Egli non fu mai il cantore delle mense plebee, i suoi gusti erano particolarmente raffinati ed il suo motto emblematico era «Comprare all'istante quanto fa comodo, senza questionare sul prezzo» (fr. 35.4).

Una tale visione del mondo (Weltanschauung) non poteva non alienargli il favore dei Cinici, ostinati custodi della morale altrui, e Cratete, una volta giunto ad Atene dalla natia Tebe, si fece un punto d'onore nel contrastare i dettami del siculo gastronomo in un'Arte culinaria, di segno completamente opposto rispetto alla liberalità del modello. Analogo anatema da parte dei filosofi del Peripato, secondo cui i simposi erano ridotti ad imbarazzanti contese fra incontinenti, che si rivolgevano le più turpi domande sulle orme di chi «sta di casa con gli scritti di Filenide e di Archestrato e passa il suo tempo a leggere la

10 Camporesi 1978, 7. 
Gastrologia di quest'ultimo» (Clearch. fr. 63 Wehrli). Né più tenero fu lo stoico Crisippo, che imputava al 'nostro' la colpa di avere corrotto i costumi dell'intera società: «... ha forse tralasciato qualcosa questo bel tomo di poeta epico di tutto ciò che può distruggere la morale, lui, unico uomo al mondo che abbia voluto emulare la vita di Sardanapalo?» (SVF III p. 199, fr. 5 v. Arnim). Eccoci al punto: la vita di spreco e la morale dell'eccesso, ma fu veramente così?

Si tratta in realtà di una contestazione strumentale e fine a se stessa, che risponde ad intenti propagandistici precisi, a sostegno di un programma politico riformatore - contrario agli eccessi del lusso (tryphé) che non poteva non travolgere il «periegeta della cucina», estremo banditore di un train de vie comme il faut.

Archestrato non era, né poteva essere un poeta-filosofo, anche se traspare in lui una qualche concessione ad istanze edonistico-epicuree, con esplicite frecciate contro i Pitagorici che disdegnano la carne dei pesci ritenuti antropofagi: «ogni pesce predilige la carne umana, se gli capiti; sicché conviene evidentemente che quanti dicono tali scempiaggini si accostino alla verdura e, al seguito del saggio Diodoro, con lui vivano in astinenza da pitagorici» (fr. 24.18-20).

Esalta continuamente l'bic et nunc, in una totale libertà di scelta. Non teme il Tartaro, a prezzo delle gioie che il cibo sa dispensare:

Lodo la focaccia che si fa ad Atene: se non riesci ad averla altrove, datti daffare, cerca di procurarti miele attico, è questo infatti che la rende superba. Così deve vivere l'uomo libero, o altrimenti, sottoterra e nel baratro, nel Tartaro, alla malora! e sia sepolto giù per stadi infiniti (fr. 60.15-20).

Nessuno scrupolo a tirare in ballo gli dèi per sottolineare la celestiale bontà di alcune vivande o anche di singoli ingredienti: è il caso del pesce-sparo,

Prendilo, quando sia tutto farcito di cacio e di olio, appendilo in forno caldo e quindi portalo bene a cottura. Cospargilo poi di sale misto a comino tritato e verde olio, facendone zampillare giù dalla mano una divina fonte (fr. 14.8-10).

O dei tranci della coda di tonno, da assumere

Belli caldi, intingendoli in pungente salamoia. Nel caso che li si voglia mangiare asciutti, sono pur sempre pregevoli, adatti per natura agli dèi immortali. / Ma se la servi cosparsa d'aceto, quella ci muore (fr. 38, 5-8). 
Tale ricchezza di spunti motiva da sola la consolidata opinione che la gastronomia archestratea fosse la metropoli dell'epicureismo e il poeta stesso la 'guida' spirituale del saggio Maestro.

Ma quali sono i gusti dell'«Esiodo o Teognide dei ghiottoni» (Ath. VII 310a)? Quale soprattutto il metodo d'indagine di questo curioso periegeta della cucina? Indubbiamente il nostro autore inaugura, sulla scia di Ananio, ma soprattutto della bistorie erodotea, un modello espositivo, destinato ben presto a divenire canonico. È lo schema archestrateo, cui si attengono di norma i ricettari più seri, che si sforzano di coniugare una originale ricerca sul campo con circostanziate istruzioni per l'uso. Di ogni cibo si indica innanzitutto la stagione migliore, poi i luoghi di elezione, per concludere - in genere - con la ricetta: QUANDO/DOVE/COME.

Il nostro gastronomo esalta una cucina tutta giocata su ingredienti di pregio (pesci rari e costosi, raffinati aromi che esaltino la qualità intrinseca dei cibi, condimenti misurati, senza alcuna concessione ad untumi e sbrodolature). La polemica più accesa è rivolta contro gli eccessi della cucina siciliana, assai celebre al tempo per varietà, ma anche proverbiale - specie quella siracusana - per eccessiva elaborazione e pesantezza. Significativa al riguardo la furiosa tirata contro i magheiroi di quelle parti:

E mentre prepari questa squisitezza (scil. il muggine-cefalo) non ti capiti fra i piedi né un Siracusano né un Italiota, non sono infatti in grado di preparare buoni pesci, ma li rovinano, imbrattando malamente ogni cosa di cacio e innaffiandola di fluido aceto e di vischiosa salamoia al silfio (fr. 46.10-17).

L'autopsia, dunque, l'osservazione personale e diretta come primario metro di giudizio - non a caso il primo verso del perduto poemetto riecheggia solennemente e scopertamente l'esordio delle Storie erodotee. Questo ricco siculo che ha girato il mondo per philedonia e si diverte a trascrivere le sue gastronomiche esperienze non ammette condizionamenti di sorta, tanto meno la sudditanza del denaro:

Quando arrivi ad Ambracia, terra beata, se vedi un pesce-capro compralo all'istante, dovessi pur pagarlo a prezzo d'oro: che la nemesi mandata dagli dèi non ti spiri contro, terribile, poiché questo pesce è veramente fior di nettare! (fr. 16.1-3).

Ed ancora: 
A Rodi, lo squalo-volpe dovessi rimetterci la pelle, nel caso non te lo vogliano vendere, rapiscilo, questa delizia che a Siracusa chiamano 'cane pingue'; poi si compia ciò che ti riserva il fato! (fr. 22).

Come Esiodo si era rivolto al fratello Perse e Teognide all'amasio Cirno, così il nostro indirizza i suoi versi agli amici Mosco e Cleaino (o Cleandro: fr. 18.3), nella canonica tradizione della poesia didascalica. Degli oltre trecento versi rimastici, solo una settantina non riguardano i pesci, questo a dimostrare il ruolo preponderante del mare e dei 'suoi figli', in tutte le manifestazioni della vita e del mondo classico. C'è da dire che il variegato panorama ittico archestrateo non risulta sempre di facile decifrazione: arduo e spesso insolubile il compito di identificare, con precise denominazioni moderne, i vari esemplari citati ${ }^{11}$.

Apre le danze l'anguilla, in particolare quella pescata nello stretto di mare di fronte a Reggio:

«Là tu, o Messinese, sei avvantaggiato su tutti gli altri mortali, perché ti metti in bocca un cibo del genere» - proclama il nostro e conclude - «insomma, a mio parere, su tutte le vivande della mensa regna sovrana e per squisitezza primeggia l'anguilla, il solo pesce, per natura, privo di genitali (fr. 10, 7-9)».

\section{L’elope si segnala a Siracusa:}

È di là, infatti, che esso trae, ancora una volta, la sua prima origine, sicché quando, in qualche modo, lo si peschi o al largo delle isole o dell'Asia o di Creta, vi arriva smilzo, tiglioso, fiaccato dalle onde (kymatoplex) (fr. 12.1-5).

L'orata si deve prendere ad Efeso, lungo le rive del venerando Selinunte, nessuna meraviglia anche se risulta di grossa pezzatura! (fr. 13).

Ancora di proporzioni dilatate lo sparo, notevole a Calcedone, non meno che a Bisanzio «pari ad uno scudo rotondo»e, come uno scudo, va appeso in «un forno bello caldo, fino a completa cottura» (fr. 14.6). Il gioco parodico risulta evidente e di sicuro effetto!

Negli anfratti dello Stretto dalle anguste correnti, non è dato trascurare la murena «cibo mirabile» (fr. 17.3), né il sinodonte di media

11 Se pure utile, non fornisce ausilio definitivo neppure il monumentale bagaglio raccolto da Thompson 1947. 
pezzatura (fr. 18), mentre a Sicione è la testa del gongro che si deve cercare e con essa «tutte le vuote parti dell'addome» (fr. 19). La predilezione degli antichi per la testa del pesce non deve stupire, essa è ricorrente anche in altri autori e, non a caso, il nostro raccomanda la testa di glauco, specie ad Olinto e a Megara (fr. 21).

Una categoria d'eccellenza fra le opzioni ittiche è rappresentata dagli squali: per lo squalo-volpe, a Rodi, bisogna esser pronti anche a lasciarci la pelle oppure a rubarlo «qualora non te lo vogliano vendere» (fr. 22.1-2).

La rassegna non conosce soste: ecco sfilare davanti ai nostri occhi la scrofa di mare, detta «arenosa scavatrice» (fr. 23), che si porta dietro, come il «cane karcharias», la fama sinistra di pesce antropofago, ma chi si affida a tali fandonie ha «anima da procellaria o da locusta e si lascia inutilmente suggestionare» (fr. 24.15-16). Ugualmente si deve tenere in non cale il gran ciarlare degli sciocchi contro l'astaco «dalle mani lunghe e pesanti ed i piedi piccoli e lenti ... i migliori per gran bontà sono a Lipari, molti ne annovera però anche l'Ellesponto» (fr. 25). A Iaso, città dei Carii, è bene procurarsi una squilla «di grandi dimensioni, rarità sul mercato» (fr. 26), mentre il citaro (varietà di rombo) ha bisogno di succulenti correttivi: è un pesce che «gode a vedere la gente scialare, incontinente qual è̀ (fr. 32.6).

Sull'eccellenza del tonno non valgono riserve: «che bisogno c'è che ti stia a dire queste cose? Non riusciresti a rovinarlo, neppure se volessi» (fr. 36.3). Il tonno di Sicilia deve essere fatto a pezzi e condito in salamoia in otri capaci (fr. 39), mentre lo sgombro va conservato per tre giorni, «prima di essere messo sotto sale, in acqua salmastra, entro un'anfora nuova».

La cavalcata fra le onde, pur con qualche affanno, potrebbe continuare, ma non vorrei scadere in una fredda elencazione che farebbe danno alla sapida leggerezza del nostro originalissimo poeta. Ciò che spero di aver fornito non è altro che un incoraggiante assaggio. Mettersi sulle tracce di un raffinato gourmet (opsologos) dell'antichità non significa necessariamente piegarsi ad una visione gourmande (opsophagos) della vita.

In definitiva, Archestato merita un'ulteriore assoluzione dalla non sempre benevola campagna di stampa, promossa paradossalmente proprio dal primo e solo paladino dei suoi versi. Vale a dire, quell'Ateneo di Naucrati, cui si devono tutti i frammenti della Vita di delizie, altrimenti passata sotto silenzio da parte degli Antichi. L'unico 
testimone non gli risparmia epiteti del tipo «centurione dei banchetti» (lochagos), «Dedalo delle mense» (opsodaidalos), «dai molti saperi» (polyistor), «saggio dei saggi», ma lo bolla anche con meno benevoli «spilorcio»(kimbix), «crapulone»(tenthes), che diverrà la qualifica peculiare, presso i moderni. Eppure, ci preme ancora una volta sottolineare, egli è - semmai - un raffinato buongustaio, quanto mai preoccupato del decoro e della forma, attento interprete di un misurato stile di vita. Un esempio fra tutti. La sua tavola deve essere per tre o quattro, al massimo cinque persone, altrimenti si trasforma in una «mensa da campo di soldatacci che vivono di rapina» (fr. 4, 3-4). In altri termini, un «gastronomo educato», secondo il felice titolo di un libro di cucina il cui autore, ignaro all'apparenza dell'illustre predecessore, scriveva nella prefazione:

Litaliano, congenitamente provinciale e conformista per pigrizia mentale, anche nel campo della gastronomia evita di uscire dall'àmbito delle sue esperienze quotidiane. Questa mancanza di curiosità è esiziale alla formazione di un vero gastronomo. ${ }^{12}$

Per essere tale, egli dice, bisogna essere «signore del mondo» e la scienza della cucina deve assurgere ad un valore universale, di eterna ricerca, di inesauste esperienze. Sembra di avere nelle orecchie l'eco lontana dei precetti archestratei.

«C'è gente che si compiace di esaltare i prodotti della propria terra» lamenta Archestrato, non senza stupore (fr. 59.20), mentre arroga per sé la libertà di considerare migliori i tonni di Ipponio rispetto a quelli siculi, in genere pescati «fuori stagione» (fr. 35.11-13). Non diversamente, il moderno gastronomo annota con sorridente verve: «Vi sono intenditori che rifiutano di uscire dalla propria abitazione, e dichiarano di apprezzare solamente le pietanze preparate dalla propria consorte o dalle proprie figlie». Che matta bestialità! Che miope oscurantismo. La totale negazione di un'arte, che non teme la prova del campo.

Ma non è questo il caso del giramondo di Gela, anzi di Catagela, secondo la spumeggiante ed icastica facezia aristofanea (Ach. 606). Come sempre è meglio imparare a sorridere, piuttosto che strozzarsi in una clamorosa risata. È, ancora una volta, questione di stile.

12 Denti di Pirajno 1964, 8. 


\section{BIBLIOGRAFIA}

BRANDT 1888 - P. Brandt, Parodorum Epicorum Graecorum et Archestrati reliquiae, Lipsiae, in Aedibus B. G. Teubneri, 1888.

CAMPORESI 1978 - P. Camporesi, Il paese della fame, Bologna, Il Mulino, 1978.

DEGANI 1973/1974 - E. Degani, Ipponatte parodico, MCr 8/9 (1973/1974), 141-167.

Degani 1975 - E. Degani, Note ai parodi greci, Sileno 1 (1975), 157-174, ora in E. Degani (a cura di), Poesia parodica greca, Bologna, Clueb, 1982.

Degani 1982 - E. Degani, Appunti di poesia gastronomica greca, in AA.VV., Prosimetrum e Spoudogeloion, Genova, Istituto di filologia classica e medievale, 1982.

Degani 1982/1983 - E. Degani, Assaggi di poesia gastronomica greca, QuadFoggia 2/3 (1982/1983), 93-112.

Degani 1991 - E. Degani, La poesia gastronomica greca, Alma Mater Studiorum 4, 1 (1991), 155.

Denti Di Pirajno 1964 - A. Denti di Pirajno, Il gastronomo educato, Vicenza, N. Pozza, 1964.

KERÉNYI 1979 - K. Kerényi, Miti e misteri, Torino, Universale scientifica Boringhieri, 1979.

OlIVIERI 1939 - A. Olivieri, Archestrato di Gela e la commedia, Dioniso 7 (1939), 110117.

Olson-SEns 2000 - S.D. Olson-Alexander Sens, Archestratos of Gela. Greek culture and cuisine in the fourth century BCE, Oxford, Oxford University Press, 2000.

RAPP 1955 - A. Rapp, The father of western gastronomy, CJ 51, 1 (1955), 43-48.

RibBeck 1877 - W. Ribbeck, Archestrati Syracusii Sive Gelensis Quae Feruntur Apud Athenaeum Reliquiae, Berolini, G. Langenscheidt, 1877.

SCINÀ 1823 - D. Scinà, I frammenti della Gastronomia, Palermo, Reale Stamperia, 1823.

Thompson 1947 - D.W. Thompson, Glossary of greek fishes, London, Oxford University Press, 1947. 